\title{
Contribution of medical education to rural health
}

Roger Y. Wong, BMSc, MD, FRCPC, FACP, FCAHS ${ }^{1}$

Author Affiliations:

1. University of British Columbia, Vancouver, Canada

The authors have no financial disclosures to declare and no conflicts of interest to report.

\section{Corresponding Author:}

Roger Y. Wong, BMSc, MD, FRCPC, FACP, FCAHS

Executive Associate Dean, Education

Clinical Professor, Department of Medicine

Faculty of Medicine

University of British Columbia

Vancouver, Canada

Email: roger.wong@ubc.ca 


\section{Abstract}

Rural health is an important priority in many jurisdictions as an example of social accountability. The choice to practice in a rural community can be influenced by personal factors, educational factors, and systemic factors. Medical education makes significant contribution to rural health by proactively and positively modifying the educational factors. The experience of the Faculty of Medicine at the University of British Columbia, Canada is used to illustrate how this can be accomplished. A multi-component approach that is tailored to address the personal and systemic determinants of rural practice must also be developed to maximize the positive impact of medical education on rural health.

\section{Keywords}

medical education, rural health 
Globally many faculties of medicine have placed increasing emphasis on social accountability, a concept that has been exemplified by the World Health Organization as "the obligation to direct their education, research and service activities towards addressing the priority health concerns of the community, region, and/or nation they have a mandate to serve ${ }^{1}$." In a number of geographically-vast jurisdictions, such as in North America, Australia, Europe and Asia, improving access to and quality of health services in rural communities is often considered as a priority within the framework of social accountability. The priority of rural health is reflected In the Canadian medical education system in at least two ways. The Future of Medical Education in Canada Medical Doctor Education (FMEC MD) project identified its top recommendation as the need to address individual and community needs ${ }^{2}$, while the Future of Medical Education in Canada Postgraduate (FMEC PG) project identified its top recommendation as the need to ensure the right mix, distribution, and number of physicians to meet societal needs ${ }^{3}$. Both of these recommendations highlight the contribution of medical education to serving the health needs of rural communities in Canada.

There are three categories of factors that influence a doctor's choice to practice in a rural community: personal factors, educational factors, and systemic factors. Among the personal factors, the most well recognized factor is the presence of rural origin, that is, previously lived experience in a rural community. ${ }^{4-6}$ Other important personal factors include career opportunity for the spouse/life partner, medical practice preference (for instance, the breadth and variety of a rural medical practice), and lifestyle preference in a smaller community, to name a few.

Educational factors are about professional development, career and personal education. These factors span the entire trajectory of medical training, from undergraduate medical education to postgraduate residency training to continuous professional development during independent practice. Examples of educational factors that can influence the choice of rural medical practice include the rural location of education and training (geographical distribution), early and recurrent exposure to different types of health care in rural communities (from small-town clinics to larger tertiary care hospitals), and clinical rotations and electives in rural health (rotational distribution).

Systemic factors include working condition (for instance, flexible work schedule, staffing level), compensation (both the amount and how it is structured), financial incentive, accommodation and living condition. Many of these factors are directly or indirectly determined by governmental and/or health organizational policies. Systemic factors play an important role not only during recruitment of a rural doctor, but also the retention of the doctor over time.

At the University of British Columbia (UBC), our experience indicates that medical education can make a significant contribution to rural health by proactively and positively modifying the educational factors. The UBC faculty of medicine, the only medical school in the Canadian province of British Columbia, underwent significant expansion and distribution of its undergraduate and postgraduate medical education programs in close partnership with the government of British Columbia, academic partners (University of Victoria, University of Northern British Columbia), and all six health authorities in the province. Since expansion began in 2004, we have more than doubled enrolment in our undergraduate and postgraduate medical programs, and simultaneously distributed medical education, clinical training, health sciences research and teaching across the province of British Columbia. The undergraduate medical 
education program comprises of four geographically distinct regional sites across the province (Vancouver-Fraser medical program with 192 students, Northern medical program with 32 students, Island medical program with 32 students, Southern medical program with 32 students). The postgraduate residency programs (72 in total and all fully accredited) have also undergone significant expansion and distribution, with the establishment of 18 new residency training sites across the province between 2002 and 2016. The goal of distributing medical education throughout the province is to prepare future doctors for the challenges and benefits of medical practice in a variety of communities, including rural, remote, northern and other underserved communities, and to encourage trainees to consider practicing in these communities upon completion of training. In addition, we work closely with the government and all six health authorities to align medical education with physician human resources planning based on population health needs and health authority service delivery plans.

There is another important learning in social accountability as we implemented at UBC what was essentially the first fully distributed medical education program of its order of magnitude in North America. We not only ensure that the training of doctors can keep up with the population needs and demographic shifts, but we also strive to achieve diversity among our medical learners that reflect the diversity of our communities, so that our learners are empowered with the skills and abilities to successfully practice in rural communities and serve diverse populations such as aboriginal communities. One example is the rural admission stream of the undergraduate medical program. We encourage medical applicants who come from or are interested in learning and practicing medicine in rural, remote, and/or northern communities. We have designed a process to assess each applicant's suitability for rural health training. A second example is the health care travelling roadshow, which is developed in conjunction with the University of Northern British Columbia, Northern Medical Programs Trust, and Rural Education Action Plan to increase the number of students who are inclined to practice in rural communities. Every year, UBC medical students travel to rural areas of the province to raise awareness among rural youth and parents about opportunities in health professions (including medicine, physical therapy, occupational therapy, midwifery). In May 2016, the travelling roadshow visited Haida Gwaii, Prince Rupert, Merritt, Princeton, and Keremeos, all of which are located in rural British Columbia. A third example is the aboriginal admissions program of the UBC undergraduate medical program. This program is strategically developed to encourage and empower prospective aboriginal students to consider a career in medicine by offering them with pre-admissions and admissions workshops, counselling support and peer mentoring opportunities early on. Since its inception, this UBC program has become a model for other Canadian faculties of medicine, and we are training more aboriginal physicians than ever before. In 2002, the aboriginal admissions program set a goal of graduating 50 more aboriginal doctors by 2020. In 2015, we were encouraged to learn that this goal was reached five years early.

While medical training takes time (6 to 10 years from the beginning of medical school to completion of residency training), we have learned from our experience over the past decade that medical education can exert a positive impact on rural health. A corollary learning is that a fulsome program evaluation plan must be developed a priori. The impact of medical education on rural health can be measured in three ways. 
Firstly, the UBC undergraduate and postgraduate medical education programs contribute to rural doctors in the province of British Columbia by increasing the overall supply of doctors. This is made possible through an increase in the entry-level undergraduate medical (baseline 122 to currently 288) and postgraduate residency (baseline 118 to currently 346) positions, an increase in the absolute number of UBC medical students remaining at UBC for residency training (from 71 in 2003 to 134 in 2010), and a more than two-fold increase in the number of residents completing training each year (395 residents completed training and were eligible to enter independent practice in 2015, among whom 180 were family physicians). Notwithstanding medical doctors are mobile to train across Canada, we observe a net gain of UBC trained doctors (from both the undergraduate and postgraduate programs) remaining in British Columbia to practice. Approximately two-thirds of trainees who completed part of their training at UBC will remain to practice in the province.

Secondly, the UBC medical education programs enhance health care delivery and capacity by increasing the supply of primary care family physicians. We observed a three-fold increase in the number of entry-level residents entering family medicine (50 residents in 2000 to 169 residents in 2016). Our family medicine residency program is the largest in Canada, and is also the most geographically distributed (delivery in 19 locations). Of note, family medicine also features vastly expanded seats for international medical graduates (baseline 12 seats to currently 52 seats).

Thirdly, and interestingly, we observe that UBC trained doctors are training and practicing in rural communities where their own training took place. Admittedly longitudinal data is still being compiled, but our early data reveals promising association between distribution of medical education and rural practice. For instance, of the medical graduates who were trained in the Northern medical program and subsequently entered independent practice in the province, 32/50 or $64 \%$ currently practice in rural communities. Another interesting observation shows that thirty percent (259/862) of British Columbia based family physicians who attended UBC for residency are practicing in rural communities. Further data is needed to see if these early observations are sustainable.

Thus far, we have described the contribution of medical education to rural health, at least from the perspective of optimizing educational factors. Universities and faculties of medicine are known to innovate and implement educational interventions, such as the UBC innovations delineated in this article. We should remember, however, that neither personal factors nor systemic factors that drive rural practice are commonly influenced by medical education alone. A multi-pronged approach that is tailored to address the personal and systemic determinants of rural practice must be developed concurrently in order to maximize the positive impact of medical education on rural health. This approach requires full collaboration among the relevant stakeholders, including academic institutions, government, health authorities, medical association, and rural communities at large. After all, social accountability is a team sport, and in the case of rural health, a sport that produces multiple winners for those who engage actively. 


\section{References}

1. Division of Development of Human Resources for Health, World Health Organization. Defining and measuring the social accountability of medical schools. Geneva, Switzerland; 1995.

2. The Association of Faculties of Medicine of Canada. The future of medical education in Canada: A collective vision for MD education. Ottawa, Ontario: The Association; 2010.

3. The Association of Faculties of Medicine of Canada. The future of medical education in Canada: A collective vision for postgraduate medical education in Canada. Ottawa, Ontario: The Association; 2012.

4. World Health Organization. Increasing access to health workers in remote and rural areas through improved retention: Global policy recommendations. Geneva, Switzerland; 2010.

5. Grobler L, Marais BJ, Mabunda SA, Marindi PN, Reuter H, Volmink J. Interventions for increasing the proportion of health professionals practising in rural and other underserved areas. The Cochrane Library. 2009.

6. Laven G, Wilkinson D. Rural doctors and rural backgrounds: how strong is the evidence? A systematic review. Aust J Rural Health. 2003; 11: 277-284. 